\title{
Weakly nonlinear asymptotics of the $\kappa-\theta$ model of cellular flames: the Q-S equation
}

\author{
Claude-Michel Brauner ${ }^{\dagger}$ \\ Mathématiques Appliquées de Bordeaux, Université Bordeaux I, 33405 Talence Cedex, France \\ MICHAEL FRANKEL ${ }^{*}$ \\ Department of Mathematical Sciences, Indiana University - Purdue University Indianapolis, \\ Indianapolis, IN 46202-3216, USA \\ JOSEPHUS HuLSHOF ${ }^{\S}$ \\ Faculty of Sciences, Mathematics and Computer Science division, \\ Vrije Universiteit Amsterdam, 1081 HV Amsterdam, The Netherlands \\ AND \\ GREGORY I. SIVASHINSKY YII \\ School of Mathematical Sciences, Tel Aviv University, Tel Aviv 69978, Israel
}

[Received 3 December 2003 and in revised form 18 January 2005]

\begin{abstract}
We consider a quasi-steady version of the $\kappa-\theta$ model of flame front dynamics introduced in [FGS03]. In this case the mathematical model reduces to a single integro-differential equation. We show that a periodic problem for the latter equation is globally well-posed in Sobolev spaces of periodic functions. We prove that near the instability threshold the solutions of the equation are arbitrarily close to these of the Kuramoto-Sivashinsky equation on a fixed time interval if the evolution starts from close configurations. We present numerical simulations that illustrate the theoretical results, and also demonstrate the ability of the quasi-steady equation to generate chaotic cellular dynamics.
\end{abstract}

2000 Mathematics Subject Classification: 35K55, 35B25, 80A25.

Keywords: Combustion; premixed flame; Kuramoto-Sivashinsky equation; front dynamics; Sobolev spaces of periodic functions; stretched coordinates.

\section{Introduction}

The laminar flames of low-Lewis-number premixtures are known to display the so-called diffusivethermal instability responsible for the formation of a non-steady cellular structure [S83]. The real-life flames constitute rather an intricate physical system involving the fluid dynamics of the multicomponent gaseous mixture, the multistep chemical kinetics, as well as the molecular and radiative transfer. It transpires, however, that the cellular instability is quite robust against these aero-thermo-chemical complexities and may be successfully captured by a simple reaction-diffusion

\footnotetext{
${ }^{\dagger}$ E-mail: brauner@math.u-bordeaux1.fr

E-mail: mfrankel@math.iupui.edu

\&E-mail: jhulshof@few.vu.nl

II E-mail: grishas@post.tau.ac.il
} 
model comprised of only two equations: the heat equation for the system's temperature and the diffusion equation for the deficient reactant's concentration. In suitably chosen units the model reads

$$
\begin{aligned}
\Theta_{t} & =\Theta_{x x}+\Theta_{y y}+\Omega(Y, \Theta), \\
Y_{t} & =\mathrm{Le}^{-1}\left(Y_{x x}+Y_{y y}\right)-\Omega(Y, \Theta), \\
\Omega & =\frac{1}{2} \mathrm{Le}^{-1} \beta^{2} Y \exp [\beta(\Theta-1) /(\sigma+(1-\sigma) \Theta)] .
\end{aligned}
$$

Here $\Theta=\left(T-T_{u}\right) /\left(T_{a d}-T_{u}\right)$ is the scaled temperature, where $T_{u}$ and $T_{a d}$ correspond to the temperature of the unburned gas and the adiabatic temperature of combustion products respectively; $Y=C / C_{u}$ is the scaled concentration of the deficient reactant with $C_{u}$ being its value in the unburned gas; $x, y, t$ are the scaled spatiotemporal coordinates referred to $D_{t h} / U$ and $D_{t h} / U^{2}$, respectively; $D_{t h}$ is the thermal diffusivity of the mixture; $U$ is the velocity of the undisturbed planar flame; Le is the Lewis number (the ratio of thermal and molecular diffusivities); $\sigma:=T_{u} / T_{a d}$; $\beta:=T_{a}(1-\sigma) / T_{a d}$ is the Zeldovich number, assumed to be large; $T_{a}$ is the activation temperature; $\Omega$ is the scaled reaction rate, where the normalizing factor $\frac{1}{2} \mathrm{Le}^{-1} \beta^{2}$ ensures that at $\beta \gg 1$ the planar flame propagates at the velocity close to unity.

The instability occurs when the Lewis number Le falls below a certain critical value $\operatorname{Le}_{c}(\beta, \sigma)<1$. Close to the instability threshold $\left(\mathrm{Le} \lesssim \mathrm{Le}_{c}\right.$ ) the flame structure becomes almost steady and near-planar. This allows for the asymptotic separation of the spatiotemporal coordinates and, as a consequence, reduction of the effective dimensionality of the system. As a result the flame dynamics may be described by a single geometrically invariant (GI) fourth-order PDE for the flame interface. In suitably chosen units it reads (see [FS87])

$$
V_{n}=1+(\alpha-1) \kappa+\kappa_{s s} .
$$

Here $V_{n}$ is the normal velocity of the flame-interface, $\kappa$ is its curvature, $s$ is the arc-length along the curved interface, and $\alpha=\frac{1}{2} \beta(1-\mathrm{Le})$.

The coordinate-free (1.4) and especially its weakly nonlinear twin, the Kuramoto-Sivashinsky (K-S) equation (1.22) appear in a variety of physical problems typically including free interface, as, for instance, in the dynamics of the solid-liquid interface of an overcooled pure substance [F88].

However, (1.4) is not the only low-dimensional model generating cellular instability. As has been realized recently, the effect may also be covered by models based on coupled second order coordinate-free systems for the flame interface and its temperature. For example, in the adiabatic case the linear stability analysis suggests the following GI system (see [FGS03]):

$$
\begin{aligned}
V_{n} & =1+\kappa+\Theta, \\
D_{t} \Theta & =\Theta_{s s}-\alpha \kappa-\Theta .
\end{aligned}
$$

Here $D_{t} \Theta$ is the Lagrangian time derivative of the interface temperature field along the "flow" generated by the normal velocity. In the intrinsic coordinates $D_{t} \Theta$ may be written as

$$
D_{t} \Theta=\Theta_{t}+\Theta_{s} \int^{s} \kappa\left(s^{\prime}, t\right) \mathrm{d} s^{\prime} .
$$

Thus, on the one hand, as one can easily verify, the $\kappa-\theta$ model (1.5. 1.6) reduces formally to (1.4) when $\alpha$ approaches unity, while, on the other hand, including the interface temperature is likely to provide a deeper connection with the original reaction-diffusion system (1.1), (1.3). Moreover, the 
$\kappa-\theta$ model may serve as a basis for the description of the flame interaction with the background flowfield, which so far has been developed only for cellularly stable flames. The $\kappa-\theta$ model may be either derived using the appropriate gradient expansion in the intrinsic coordinates, or, as in [FGS03], may be built as a geometrically invariant extrapolation consistent with the relation provided by the linear stability analysis of the planar flame.

However, one can observe, for instance via numerical simulation, that not far from the instability threshold the time derivative in the second equation of the $\kappa-\theta$ model (1.5) 1.6) has a relatively small effect on the solution. Based on this observation one can define a quasi-steady $\kappa-\theta$ model as follows:

$$
\begin{gathered}
V_{n}=1+\kappa+\Theta, \\
\Theta_{s s}-\alpha \kappa-\Theta=0 .
\end{gathered}
$$

Indeed, as we rigorously demonstrate below for the weakly nonlinear case, the solutions of the quasi-steady model are asymptotically close to the solutions of the K-S equation.

For a mildly distorted planar flame, propagating along the $y$-axis, the flame interface may be described by an explicit function of the transversal coordinate $x$,

$$
y=-t+\Phi(x, t) .
$$

In this case,

$$
\begin{gathered}
V_{n} \simeq 1-\Phi_{t}-\frac{1}{2} \Phi_{x}^{2} \\
\kappa \simeq \Phi_{x x}, \quad \mathrm{~d} s \simeq \mathrm{d} x, \quad \text { and } \quad D_{t} \simeq \Theta_{t}+\Phi_{x} \Theta_{x} .
\end{gathered}
$$

As a result one obtains weakly nonlinear versions of the $\kappa-\theta$ models,

$$
\begin{aligned}
\Phi_{t}+\frac{1}{2} \Phi_{x}^{2} & =\Phi_{x x}-\Theta, \\
\Theta_{t}+\Phi_{x} \Theta_{x} & =\Theta_{x x}+\alpha \Phi_{x x}-\Theta,
\end{aligned}
$$

and, respectively,

$$
\begin{gathered}
\Phi_{t}+\frac{1}{2} \Phi_{x}^{2}=\Phi_{x x}-\Theta, \\
\Theta_{x x}+\alpha \Phi_{x x}-\Theta=0 .
\end{gathered}
$$

The reduced models (as well as their geometrically invariant counterparts) constitute new dynamical systems not explored yet either analytically or numerically.

It is quite obvious that the inversion

$$
\Theta=\alpha\left(I-\partial_{x}^{2}\right)^{-1} \Phi_{x x}
$$

is well defined on any reasonable function space to be considered for this problem, therefore one can think of (1.14) as a relaxation equation around the equilibrium (1.17).

The current paper is devoted to the analysis of the system 1.15, 1.16) that we rewrite as a single nonlocal equation

$$
\Phi_{t}+\frac{1}{2} \Phi_{x}^{2}=\Phi_{x x}-\alpha\left(I-\partial_{x}^{2}\right)^{-1} \Phi_{x x} .
$$

One can consider two natural boundary value problems on a finite interval for 1.18 ). One is the adiabatic problem corresponding to the flame propagation in an insulated combustible strip. 
Another one is the periodic problem corresponding to the combustion of a thin cylindrical shell. Both settings reflect the main dynamical features of the system for a sufficiently wide interval.

However, the periodic problem is somewhat more convenient for the rigorous study, as one deals with the exponentials as a basis in the Fourier representation which is invariant under differentiation. This is why in the current article we choose to study the latter. We note also that the bulk of analytical work regarding the K-S equation has been performed with the periodic conditions.

Thus, for the periodic problem on the interval $[-L / 2, L / 2]$ that we study below, $\left(I-\partial_{x}^{2}\right)^{-1}$ can be explicitly expressed (cf. Sec. 4) as

$$
\left[\left(I-\partial_{x}^{2}\right)^{-1} f\right](x)=\frac{1}{2} \int_{-L / 2}^{L / 2} \frac{\cosh (\eta) f(x-\eta-L / 2)}{\sinh (L / 2)} \mathrm{d} \eta .
$$

The basic feature of $(1.18)$ is that, in a more abstract form:

$$
\Phi_{t}+\frac{1}{2} \Phi_{x}^{2}+A \Phi-\alpha B \Phi=0,
$$

there is a competition between the positive operators $A=-\partial_{x}^{2}$ and $\alpha B, B=(I+A)^{-1} A$. This situation is reminiscent of the K-S equation, where the competition is between $A^{2}$ and $A$. Equation (1.18) turns out to be rather benign: as we show below the periodic boundary value problem for it is globally well posed.

The main objective of the paper, however, is to establish a rigorous link between the QuasiSteady Model (Q-S), namely (1.18), and the K-S equation. Indeed, about the equilibrium $\Phi=0$ of (1.18) the dispersion relation yields

$$
\omega_{k}=-k^{2}+\frac{\alpha k^{2}}{1+k^{2}}=(\alpha-1) k^{2}-\alpha k^{4}+\cdots .
$$

Setting $0<\alpha-1:=\varepsilon \ll 1$, we observe that in the long-wave range $k=O(\sqrt{\varepsilon}), 1.21$ is identical to the dispersion relation of the K-S equation [S80]. At the same time the behavior of the spectrum 1.21 for the Q-S equation (1.18) for large $k: \omega_{k} \sim-k^{2}$ is identical to that of the original freeinterface problem [MS79], in contrast to K-S where it is $\sim-k^{4}$. However, it seems plausible that the asymptotic dynamics of the front for $t=O\left(\varepsilon^{-2}\right)$ and $x=O\left(\varepsilon^{-1 / 2}\right)$ is the same as that of the K-S equation. More precisely, in the coordinates $\tau=t \varepsilon^{2}, \xi=x \sqrt{\varepsilon}$, we anticipate $\Phi \sim \varepsilon U$, where $U$ solves the K-S equation

$$
U_{\tau}+\frac{1}{2}\left(U_{\xi}\right)^{2}+U_{\xi \xi \xi \xi}+U_{\xi \xi}=0 .
$$

Using more rigorous terms, let $U$ be the periodic solution of (1.22) on a fixed time interval $\left[0, T_{0}\right]$ with initial condition $U_{0}$ of period $L_{0}>0$. We prove that, for $0<\varepsilon \leqslant \varepsilon_{0}$, the unique solution $\Phi$ of 1.18 with period $L_{0} / \sqrt{\varepsilon}$ is such that

$$
\max \left|\Phi(x, t)-\varepsilon U\left(x \sqrt{\varepsilon}, t \varepsilon^{2}\right)\right| \leqslant C \varepsilon^{2}
$$

for $0 \leqslant t \leqslant T_{0} / \varepsilon^{2}$. A basic assumption is that the initial data $\Phi_{0}$ for $\Phi$ are "well-prepared"(see e.g. [GM97, DFM02]): $\Phi_{0}=\varepsilon U_{0}+\varepsilon^{2} u_{0}$, where $u_{0}$, for simplicity, does not depend on $\varepsilon$, is sufficiently smooth and has also period $L_{0} / \sqrt{\varepsilon}$. The value $\varepsilon_{0}$ depends only on $L_{0}, T_{0}$ and initial data.

Although the quasi-steady model was introduced $a d$ hoc to serve as it were as a "bridge" between the full $\kappa-\theta$ model and the GI equation (1.4), it represents in our opinion an interesting dynamical system in its own right. The primary objective of a limited set of numerical solutions that we present 
in Section 4 was to verify at least a qualitative closeness of the solutions of the quasi-steady model to the solutions of the K-S equation even for moderately small values of $\varepsilon$, and, secondly, to show that the quasi-steady model generates cellular structure and turbulence.

We need to clarify that the asymptotic convergence to the K-S equation is in terms of the instability parameter $\varepsilon$ for a fixed albeit arbitrarily large time, and not in terms of $t \rightarrow \infty$. Since the spatial interval $L=L_{0} / \sqrt{\varepsilon}$ and the instability parameter in $(1.23$ depend on $\varepsilon \ll 1$, and the time that it takes for a given solution to approach the attractor may be extremely large, we cannot at this point make any rigorous claims concerning the final pattern that is developed, and, therefore, we need the numerical solution to demonstrate the similarity of fully developed dynamics between the Q-S equation and the K-S equation. Moreover, it is interesting to observe the behavior exhibited by the system in the deeply nonlinear range where $\varepsilon$ is not small and the result of Section 3 is not valid.

This study was also motivated by the desire to explore farther the role played by the K-S equation as a "modulation equation" in combustion, chemistry, fluid mechanics, etc. We hope that our approach can be extended to more complex models, especially to free boundary problems in combustion and dynamics of free boundaries (see [BL00, BHL01]). Yet another component of our interest in the quasi-steady equation is an attempt to understand what particular features of a nonlinear equation are important for generation of specific dynamical patterns. We hope that this and the future comparative studies of the full $\kappa-\theta$ model, the quasi-steady model, and the K-S equation may shed some light on this matter.

The paper is organized as follows: in Section 2 we check that the problem is well-posed in the framework of Sobolev spaces of $L$-periodic functions for fixed $\alpha$. We work with the differentiated equation for $\Psi=\Phi_{x}$. This is a standard trick (see the treatment of the K-S equation in [Temam]). The nonlinearity $\Psi \Psi_{x}$ is easier to handle than $\Phi_{x}^{2}$.

In Section 3 , we set $\Phi=\varepsilon \varphi$, hence $\Psi=\varepsilon^{3 / 2} \psi$, and consider the problem for $\psi$ in the stretched coordinates $\xi$ and $\tau$, with fixed space period $L_{0}$ and time interval $\left[0, T_{0}\right]$. We express $\varphi$ as $\varphi=$ $U+\varepsilon u, \psi=V+\varepsilon v$, where $U$ solves the K-S equation on $\left[-L_{0} / 2, L_{0} / 2\right] \times\left[0, T_{0}\right]$, and $V=U_{\xi}$ solves the differentiated K-S equation. We study the problem for $v$. We point out that the error estimate 1.23 is based only on the control of the $L^{2}$ norm of $v(t)$.

Finally, Section 4 is devoted to the numerical simulation. Numerical results are presented which are fully in accordance with the theoretical part. We also demonstrate numerically that the Q-S equation generates chaotic solutions on a sufficiently wide interval.

We should emphasize right away that neither the global existence results obtained in Section 2 nor the asymptotic closeness to K-S on a given time interval (Section 3) guarantee the behavior characteristic of the dissipative systems as one would naturally expect. Indeed, existence of an absorbing ball, and, consequently, of a global compact attractor of finite Hausdorff dimension, as well as a more detailed numerical study of the dynamics of the quasi-steady model will be the subjects of forthcoming papers.

\section{Mathematical setting}

Let $L>0$ and $T>0$ be fixed. We consider 1.18 ,

$$
\Phi_{t}+\frac{1}{2} \Phi_{x}^{2}=\Phi_{x x}-\alpha\left(I-\partial_{x}^{2}\right)^{-1} \Phi_{x x}
$$

on the time interval $[0, T]$ in the class of periodic functions with period $L$ in $x$. Differentiation with 
respect to $x$ leads to

$$
\Psi_{t}+\Psi \Psi_{x}=\Psi_{x x}-\alpha\left(I-\partial_{x}^{2}\right)^{-1} \Psi_{x x}
$$

for $\Psi=\Phi_{x}$ which has zero average. We easily recover $\Phi$ from $\Psi$, in view of

$$
\frac{\mathrm{d}}{\mathrm{d} t} \int_{-L / 2}^{L / 2} \Phi(x, t) \mathrm{d} x=-\frac{1}{2} \int_{-L / 2}^{L / 2} \Psi(x, t)^{2} \mathrm{~d} x .
$$

For integer or arbitrary real $s$ we denote by $H^{s}$ the usual Sobolev spaces of $L$-periodic (generalized) functions with zero average, which we will conveniently represent as

$$
H^{s}=\left\{w=\sum_{k=1}^{\infty} a_{k} w_{k}: \sum_{k=1}^{\infty} \lambda_{k}^{s} a_{k}^{2}<\infty\right\}
$$

with norm

$$
\|w\|_{s}^{2}=\sum_{k=1}^{\infty} \lambda_{k}^{s} a_{k}^{2} \quad\left(=\left|w^{(s)}\right|^{2}=\int_{-L / 2}^{L / 2} w^{(s)}(x)^{2} \mathrm{~d} x \text { if } s=0,1,2, \ldots\right) .
$$

Here the $w_{k}$ are a complete set of eigenfunctions of the operator

$$
A=-\frac{\mathrm{d}^{2}}{\mathrm{~d} x^{2}}=-\partial_{x}^{2}: D(A)=H^{2} \rightarrow H^{0}
$$

corresponding to the positive eigenvalues $\lambda_{1} \leqslant \lambda_{2} \leqslant \lambda_{3} \leqslant \cdots \uparrow \infty$. Note that the eigenvalue zero does not occur in this setting because nonzero constant functions are excluded from our Sobolev spaces.

These eigenfunctions are also eigenfunctions of the operator

$$
B=(I+A)^{-1} A: H^{0} \rightarrow H^{0},
$$

with eigenvalues $\lambda_{n} /\left(1+\lambda_{n}\right)$, so that, with Parseval's identity,

$$
\begin{gathered}
\int_{-L / 2}^{L / 2} w(x) B w(x) \mathrm{d} x=\sum_{k=1}^{\infty} \frac{\lambda_{k}}{1+\lambda_{k}} a_{k}^{2} \leqslant\|w\|_{0}^{2}=|w|^{2}=\int_{-L / 2}^{L / 2} w(x)^{2} \mathrm{~d} x, \\
\int_{-L / 2}^{L / 2}-w^{\prime \prime}(x) B w(x) \mathrm{d} x=\sum_{k=1}^{\infty} \frac{\lambda_{k}^{2}}{1+\lambda_{k}} a_{k}^{2} \leqslant\|w\|_{1}^{2}=\left|w^{\prime}\right|^{2}=\int_{-L / 2}^{L / 2} w^{\prime}(x)^{2} \mathrm{~d} x .
\end{gathered}
$$

Moreover,

$$
A_{\alpha}=A-\alpha B: D\left(A_{\alpha}\right)=H^{2} \rightarrow H^{0} \quad \text { has } \quad A_{\alpha} w_{n}=\lambda_{n}\left(1-\frac{\alpha}{1+\lambda_{n}}\right) w_{n},
$$

and therefore

$$
\int_{-L / 2}^{L / 2} w(x) A_{\alpha} w(x) \mathrm{d} x=\sum_{k=1}^{\infty}\left(\left(\lambda_{k}+\frac{1-\alpha}{2}\right)^{2}-\left(\frac{1-\alpha}{2}\right)^{2}\right) \frac{a_{k}^{2}}{1+\lambda_{k}} .
$$


If we denote the map $\Psi \mapsto \Psi \Psi_{x}$ by $F$, then the abstract form of (2.24) reads

$$
\Psi_{t}+A_{\alpha} \Psi+F(\Psi)=0 .
$$

Clearly $A_{\alpha}$ defines a sectorial operator. Since any intermediate space $H^{s}$ with $0<s<2$ is an interpolation space, local well-posedness for initial data in $H^{2}$ follows by analytic semigroup methods, provided we can choose $s$ such that $F: H^{s} \rightarrow H^{0}$ is locally Lipschitz. With $H^{1}$ embedding in $L^{\infty}$, we may choose $s=1$.

To establish global existence we observe first that if we represent $\Psi$ as a Fourier series

$$
\Psi=\sum_{k=1}^{\infty} a_{k}(t) w_{k}
$$

and multiply by $\Psi$, then integration yields, in view of 2.26, ,

$$
\frac{1}{2} \frac{\mathrm{d}}{\mathrm{d} t} \int_{-L / 2}^{L / 2} \Psi^{2} \mathrm{~d} x+\sum_{k=1}^{\infty}\left(\lambda_{k}+\frac{1-\alpha}{2}\right)^{2} \frac{a_{k}(t)^{2}}{1+\lambda_{k}} \leqslant \sum_{k=1}^{\infty}\left(\frac{1-\alpha}{2}\right)^{2} \frac{a_{k}(t)^{2}}{1+\lambda_{k}} \leqslant \frac{\beta}{2} \int_{-L / 2}^{L / 2} \Psi^{2} \mathrm{~d} x,
$$

where $\beta=(1-\alpha)^{2}$ appears as a characteristic time, whence

$$
\max _{0 \leqslant t \leqslant T}\|\Psi(t)\|_{0}^{2}+2 \int_{0}^{T} \sum_{k=1}^{\infty}\left(\lambda_{k}+\frac{1-\alpha}{2}\right)^{2} \frac{a_{k}(t)^{2}}{1+\lambda_{k}} \mathrm{~d} t \leqslant\|\Psi(0)\|_{0}^{2} \exp (\beta T) .
$$

We conclude that

$$
\max _{0 \leqslant t \leqslant T}\|\Psi(t)\|_{0}^{2}+C \int_{0}^{T}\|\Psi(t)\|_{1}^{2} \mathrm{~d} t \leqslant\|\Psi(0)\|_{0}^{2} \exp (\beta T)
$$

for some positive constant $C$, which takes into account that

$$
\left(\lambda_{k}+\frac{1-\alpha}{2}\right)^{2} \frac{1}{1+\lambda_{k}} \sim \lambda_{k}
$$

only as $k \rightarrow \infty$. Moreover, thanks to $H^{-1} \supset L^{1}$ (with zero average),

$$
\int_{0}^{T}\left\|\Psi \Psi_{x}\right\|_{-1}^{2} \mathrm{~d} t \leqslant C\left(\max _{0 \leqslant t \leqslant T}\|\Psi(t)\|_{0}^{2}\right) \int_{0}^{T}\|\Psi(t)\|_{1}^{2} \mathrm{~d} t \leqslant C\|\Psi(0)\|_{0}^{4} \exp (2 \beta T) .
$$

Estimates 2.28, 2.29) allow the construction of a weak solution satisfying 2.28, 2.29) for initial data $\Psi(0)$ in $H^{0}$, and a variant of 2.29 gives uniqueness.

Next we observe that for $t>0$ the solutions are in fact smooth, as long as they exist. This follows from standard estimates for solutions of $u_{t}+A_{\alpha} u=f(t)$, which are easily derived using the differential equations for the Fourier coefficients. Representing $u$ and $f$ as

$$
u=\sum_{k=1}^{\infty} a_{k}(t) w_{k}, \quad f=\sum_{k=1}^{\infty} b_{k}(t) w_{k},
$$


we have

$$
\dot{a}_{k}+\mu_{k} a_{k}=b_{k}, \quad \mu_{k}=\lambda_{k}\left(1-\frac{\alpha}{\lambda_{k}+1}\right) \sim \lambda_{k},
$$

whence, for $\mu_{k}>0$,

$$
a_{k}(T)^{2}+\int_{0}^{T} \mu_{k} a_{k}(t)^{2} \mathrm{~d} t \leqslant a_{k}(0)^{2}+\int_{0}^{T} \frac{b_{k}(t)^{2}}{\mu_{k}} \mathrm{~d} t
$$

and also,

$$
a_{k}(T)^{2}+\int_{0}^{T} \frac{\dot{a}_{k}(t)^{2}}{\mu_{k}} \mathrm{~d} t \leqslant a_{k}(0)^{2}+\int_{0}^{T} \frac{b_{k}(t)^{2}}{\mu_{k}} \mathrm{~d} t .
$$

Note that for $\alpha>0$ there will be a finite number of negative eigenvalues $\mu_{k}$, which will make the corresponding Fourier coefficients grow, but those are easily controlled by a $T$-dependent constant. It thus follows by standard procedures that

$$
\begin{aligned}
\max _{0 \leqslant t \leqslant T}\|u(t)\|_{s}^{2}+\int_{0}^{T}\|u(t)\|_{s+1}^{2} \mathrm{~d} t+\int_{0}^{T}\|\dot{u}(t)\|_{s-1}^{2} \mathrm{~d} t & \\
& \leqslant C\left(\|u(0)\|_{s}^{2}+\int_{0}^{T}\|f(t)\|_{s-1}^{2} \mathrm{~d} t\right),
\end{aligned}
$$

where the constant $C$ depends on $\alpha, s, L$ and $T$. Employing this estimate with $s=0$ is consistent with 2.28, 2.29 but does not improve the regularity of $\Psi$.

Since

$$
\|u v\|_{s} \leqslant C\|u\|_{s}\|v\|_{s} \quad(s>1 / 2)
$$

we have, with changing constants,

$$
\begin{aligned}
\int_{0}^{T}\left\|u u_{x}\right\|_{s}^{2} \mathrm{~d} t & \leqslant C \max _{0 \leqslant t \leqslant T}\|u(t)\|_{s}^{2} \int_{0}^{T}\left\|u_{x}\right\|_{s}^{2} \mathrm{~d} t \\
& \leqslant C\left(\max _{0 \leqslant t \leqslant T}\|u(t)\|_{s}^{2}\right) \int_{0}^{T}\|u(t)\|_{s+1}^{2} \mathrm{~d} t .
\end{aligned}
$$

For the local solution $\Psi$ above with initial data in $D\left(A_{\alpha}\right)=H^{2}$, the estimates 2.30, 2.31 allow a successful bootstrap argument local in time, to show that the solution is smooth, as long as it exists.

To get a global bound however, such as

$$
\max _{0 \leqslant t \leqslant T}\|\Psi(t)\|_{s}^{2}+\int_{0}^{T}\|\Psi(t)\|_{s+1}^{2} \mathrm{~d} t \leqslant C,
$$

where $C$ is allowed to depend on $\alpha, s, L$ and $T$ of course, but otherwise only on the initial data through $\|\Psi(0)\|_{s}$, we have to start from a global estimate. With 2.31) invalid for $s=0$, we need a second uniform estimate. If we multiply the equation by $-\Psi_{x x}$, then integration yields

$$
\frac{1}{2} \frac{\mathrm{d}}{\mathrm{d} t} \int_{-L / 2}^{L / 2} \Psi_{x}^{2} \mathrm{~d} x+\int_{-L / 2}^{L / 2} \Psi_{x x}^{2} \mathrm{~d} x \leqslant \alpha \int_{-L / 2}^{L / 2} \Psi_{x}^{2} \mathrm{~d} x+\int_{-L / 2}^{L / 2} \Psi \Psi_{x} \Psi_{x x} \mathrm{~d} x
$$


The last term on the right hand side is estimated by

$$
\begin{aligned}
\int_{-L / 2}^{L / 2} \Psi \Psi_{x} \Psi_{x x} \mathrm{~d} x & \leqslant \frac{1}{2} \int_{-L / 2}^{L / 2} \Psi_{x x}^{2} \mathrm{~d} x+\frac{1}{2} \max _{x}|\Psi(x)|^{2} \int_{-L / 2}^{L / 2} \Psi_{x}^{2} \mathrm{~d} x \\
& \leqslant \frac{1}{2} \int_{-L / 2}^{L / 2} \Psi_{x x}^{2} \mathrm{~d} x+\frac{L}{2}\left(\int_{-L / 2}^{L / 2} \Psi_{x}^{2} \mathrm{~d} x\right)^{2}
\end{aligned}
$$

which combined with the previous estimate gives

$$
\frac{\mathrm{d}}{\mathrm{d} t} \int_{-L / 2}^{L / 2} \Psi_{x}^{2} \mathrm{~d} x+\int_{-L / 2}^{L / 2} \Psi_{x x}^{2} \mathrm{~d} x \leqslant\left(2 \alpha+L \int_{-L / 2}^{L / 2} \Psi_{x}^{2} \mathrm{~d} x\right) \int_{-L / 2}^{L / 2} \Psi_{x}^{2} \mathrm{~d} x .
$$

By (2.28) the last factor on the right hand side is integrable on [0, T]. Consequently, 2.32) holds with $s=1$ for the semigroup solution $\Psi$ with initial data $\Psi(0)$ in $H^{2}$, but the constant depends only on $\|\Psi(0)\|_{1}$. Taking limits one then easily constructs global weak solutions for initial data $\Psi(0)$ in $H^{1}$, satisfying the same bound, i.e. 2.32 with $s=1$.

As for the global bootstrap argument, we note that (2.32) with $s=1$ and $(2.31)$ imply that with $\Psi(0)$ in $H^{2}$, 2.32) holds with $s=2$. If also $\Psi(0)$ is in $H^{3}$, then 2.32) with $s=2$ and 2.31 imply that (2.32) holds with $s=3$, and so on. Thus the bootstrap gives (2.32) with any $s \geqslant 1$, provided $\Psi(0)$ is in $H^{s}$.

The consequences are stated in the following theorem.

THEOREM 2.1 Let $\Psi_{0}$ be given in $H^{s}$ where $s=0,1,2,3, \ldots$ Then 2.24 has a unique solution on any time interval $[0, T]$ with $\Psi=\Psi_{0}$ at $t=0$. The solution belongs to $L^{2}\left(0, T ; H^{s+1}\right) \cap$ $C^{0}\left([0, T] ; H^{s}\right)$, and has $\Psi_{t} \in L^{2}\left(0, T ; H^{s-1}\right)$, and the corresponding norms are bounded by a constant which depends only on $\alpha, s, L, T$ and $\|\Psi(0)\|_{s}$. Thus the solution is smooth (and in fact analytic in time) for $t>0$. Therefore

$$
\left(I-\partial_{x}^{2}\right)\left(\Psi_{t}+\Psi \Psi_{x}\right)+\Psi_{x x x x}+(\alpha-1) \Psi_{x x}=0
$$

for $t>0$. The latter equation holds in $L^{2}\left(0, T ; H^{0}\right)$ as soon as $s=3$. For 1.18 the statement is similar, with initial data $\Phi_{0}$ in $H^{s}$, where $s=1,2,3, \ldots$, and (1.18) rewritten as

$$
\left(I-\partial_{x}^{2}\right)\left(\Phi_{t}+\frac{1}{2} \Phi_{x}^{2}\right)+\Phi_{x x x x}+(\alpha-1) \Phi_{x x}=0 .
$$

Next we consider the same issues for the K-S equation

$$
\Phi_{t}+\frac{1}{2} \Phi_{x}^{2}+\Phi_{x x}+\Phi_{x x x x}=0
$$

on the time interval $[0, T]$ in the class of periodic functions with period $L$ in $x$. Differentiation with respect to $x$ then leads to

$$
\Psi_{t}+\Psi \Psi_{x}+\Psi_{x x x x}+\Psi_{x x}=0
$$

for $\Psi=\Phi_{x}$ which has zero average [Temam, NS84].

The eigenfunctions $w_{n}$ are also eigenfunctions of the operator $A^{2}-A: D\left(A^{2}\right)=H^{4} \rightarrow H^{0}$, with eigenvalues $\mu_{k}=\lambda_{k}\left(\lambda_{k}-1\right)$, and the abstract form of 2.36) reads

$$
\Psi_{t}+\left(A^{2}+A\right) \Psi+F(\Psi)=0 .
$$


Local well-posedness for initial data in $H^{4}$ follows as before by analytic semigroup methods. Note that

$$
\int_{-L / 2}^{L / 2} w(x)\left(A^{2}+A\right) w(x) \mathrm{d} x=\sum_{k=1}^{\infty}\left(\left(\lambda_{k}-\frac{1}{2}\right)^{2}-\frac{1}{4}\right) a_{k}^{2} .
$$

If we multiply the equation by $\Psi$, then integration yields, in view of 2.38,

$$
\frac{1}{2} \frac{\mathrm{d}}{\mathrm{d} t} \int_{-L / 2}^{L / 2} \Psi^{2} \mathrm{~d} x+\sum_{k=1}^{\infty}\left(\lambda_{k}-\frac{1}{2}\right)^{2} a_{k}(t)^{2} \leqslant \frac{1}{4} \sum_{k=1}^{\infty} a_{k}(t)^{2}=\frac{1}{4} \int_{-L / 2}^{L / 2} \Psi^{2} \mathrm{~d} x,
$$

whence

$$
\max _{0 \leqslant t \leqslant T}\|\Psi(t)\|_{0}^{2}+C \int_{0}^{T}\|\Psi(t)\|_{2}^{2} \mathrm{~d} t \leqslant\|\Psi(0)\|_{0}^{2} \exp \left(\frac{T}{2}\right)
$$

for some positive constant $C$.

Estimates for solutions of $u_{t}+\left(A^{2}-A\right) u=f(t)$ now come from

$$
\dot{a}_{k}+\mu_{k} u_{k}=b_{k}, \quad \mu_{k}=\lambda_{k}\left(\lambda_{k}-1\right) \sim \lambda_{k}^{2},
$$

and provide us with

$$
\max _{0 \leqslant t \leqslant T}\|u(t)\|_{s}^{2}+\int_{0}^{T}\|u(t)\|_{s+2}^{2} \mathrm{~d} t \leqslant C\left(\|u(0)\|_{s}^{2}+\int_{0}^{T}\|f(t)\|_{s-2}^{2} \mathrm{~d} t\right),
$$

where the constant depends on $\alpha, s, L$ and $T$.

With initial data in $H^{1}$, estimate 2.29 is enough to start the global bootstrap, which may then be continued with 2.31 as before. Omitting further details we state the result in the following theorem.

THEOREM 2.2 Let $\Psi_{0}$ be given in $H^{s}$ where $s=0,1,2,3, \ldots$ Then 2.37) has a unique solution on any time interval $[0, T]$ with $\Psi=\Psi_{0}$ at $t=0$. The solution belongs to $L^{2}\left(0, T ; H^{s+2}\right) \cap$ $C^{0}\left([0, T] ; H^{s}\right)$, and has $\Psi_{t} \in L^{2}\left(0, T ; H^{s-2}\right)$. The solution is smooth for $t>0$. For 2.35 the statement is similar, with initial data $\Phi_{0}$ in $H^{s}$, where $s=1,2,3, \ldots$

\section{Asymptotics}

In this section, we are interested in $\alpha=1+\varepsilon$, where $\varepsilon$ is a (small) fixed positive number. In fact $\varepsilon^{2}$ is nothing other than $\beta$ in 2.28 . Moreover (a key point), we take the period to depend on $\varepsilon$, introducing a reference period $L_{0}>0$ and time interval $\left[0, T_{0}\right]$. The reference space and time variables will be denoted by $\xi$ and $\tau$. We consider (1.18), equivalently written as (2.34), in the class of periodic functions with period $L_{0} / \sqrt{\varepsilon}$ in $x$, on the time interval $\left[0, T_{0} / \varepsilon^{2}\right]$ in $t$.

We rescale

$$
\Phi=\varepsilon \varphi, \quad t=\tau / \varepsilon^{2}, \quad x=\xi / \sqrt{\varepsilon},
$$

whence (2.34) becomes

$$
\left(I-\varepsilon \partial_{\xi}^{2}\right)\left(\varphi_{\tau}+\frac{1}{2} \varphi_{\xi}^{2}\right)+\varphi_{\xi \xi \xi \xi}+\varphi_{\xi \xi}=0 .
$$


We then look for $\varphi$ as

$$
\varphi=U+\varepsilon u,
$$

so that with $\varepsilon=0$ in 3.41 , we recover the 4 th order K-S equation

$$
U_{\tau}+\frac{1}{2} U_{\xi}^{2}+U_{\xi \xi \xi \xi}+U_{\xi \xi}=0
$$

Our aim now is to establish a uniform bound on $u$ which is valid for small positive $\varepsilon$, uniformly in $\varepsilon$. For $u$ the equation reads

$$
\left(I-\varepsilon \partial_{\xi}^{2}\right)\left(u_{\tau}+\frac{1}{2}\left(2 U_{\xi} u_{\xi}+\varepsilon u_{\xi}^{2}\right)\right)+u_{\xi \xi \xi \xi}+u_{\xi \xi}=\partial_{\xi}^{2}\left(U_{\tau}+\frac{1}{2} U_{\xi}^{2}\right) .
$$

As in Section 2 we work with the differentiated equations for $\psi=\phi_{\xi}, V=U_{\xi}, v=u_{\xi}$, which are

$$
\begin{gathered}
\left(I-\varepsilon \partial_{\xi}^{2}\right)\left(\psi_{\tau}+\psi \psi_{\xi}\right)+\psi_{\xi \xi \xi \xi}+\psi_{\xi \xi}=0, \\
V_{\tau}+V V_{\xi}+V_{\xi \xi \xi \xi}+V_{\xi \xi}=0, \\
\left(I-\varepsilon \partial_{\xi}^{2}\right)\left(v_{\tau}+(V v)_{\xi}+\varepsilon v v_{\xi}\right)+v_{\xi \xi \xi \xi}+v_{\xi \xi}=\partial_{\xi}^{2}\left(V_{\tau}+V V_{\xi}\right) .
\end{gathered}
$$

Note that

$$
\Psi=\varepsilon^{3 / 2} \psi, \quad \psi=V+\varepsilon v,
$$

and that $u$ is recovered from $v$ since its mean value $\bar{u}$ satisfies

$$
\bar{u}_{\tau}+\frac{1}{2 L_{0}} \int_{-L_{0} / 2}^{L_{0} / 2} v V \mathrm{~d} \xi+\varepsilon^{2} \int_{-L_{0} / 2}^{L_{0} / 2} v^{2} \mathrm{~d} \xi=0 .
$$

For $\varphi$ we now consider so-called "well-prepared" $L_{0}$-periodic initial data of the form

$$
\varphi(\xi, 0)=\varphi_{0}(\xi)=U_{0}(\xi)+\varepsilon u_{0}(\xi)
$$

whence, with $\psi_{0}=\phi_{0}^{\prime}, V_{0}=U_{0}^{\prime}, v_{0}=u_{0}^{\prime}$, for $\psi$,

$$
\psi(\xi, 0)=\psi_{0}(\xi)=V_{0}(\xi)+\varepsilon v_{0}(\xi)
$$

Thus $U_{0}, V_{0}, u_{0}, v_{0}$ are the initial data for $U, V, u, v$.

The basic estimate in this section comes from testing (3.46) by $v$, which with the usual notation $|v|^{2}:=\int_{-L_{0} / 2}^{L_{0} / 2} v(\xi)^{2} \mathrm{~d} \xi$ yields

$$
\begin{array}{rl}
\frac{1}{2} \frac{\mathrm{d}}{\mathrm{d} \tau}\left(|v|^{2}+\varepsilon\left|v_{\xi}\right|^{2}\right)+\int_{-L_{0} / 2}^{L_{0} / 2}(V v)_{\xi} v & \mathrm{~d} \xi-\varepsilon \int_{-L_{0} / 2}^{L_{0} / 2}\left((V v)_{\xi}+\varepsilon v v_{\xi}\right) v_{\xi \xi} \mathrm{d} \xi \\
& +\left|v_{\xi \xi}\right|^{2}-\left|v_{\xi}\right|^{2}=\int_{-L_{0} / 2}^{L_{0} / 2}\left(V_{\tau}+V V_{\xi}\right) v_{\xi \xi} \mathrm{d} \xi .
\end{array}
$$

Writing also $|v|_{\infty}:=\sup _{\xi}|v(\xi)|$ we have

$$
\begin{aligned}
\frac{1}{2} \frac{\mathrm{d}}{\mathrm{d} \tau}\left(|v|^{2}+\varepsilon\left|v_{\xi}\right|^{2}\right) & +\left|v_{\xi \xi}\right|^{2} \leqslant|V|_{\infty}\left|v_{\xi}\right||v|+\left|V_{\xi}\right|_{\infty}|v|^{2}+\varepsilon|V|_{\infty}\left|v_{\xi}\right|\left|v_{\xi \xi}\right| \\
& +\varepsilon\left|V_{\xi}\right|_{\infty}|v|\left|v_{\xi \xi}\right|+\varepsilon^{2}|v|_{\infty}\left|v_{\xi}\right|\left|v_{\xi \xi}\right|+\left|v_{\xi}\right|^{2}+\left|V_{\tau}+V V_{\xi}\right|\left|v_{\xi \xi}\right|
\end{aligned}
$$


Then, in view of $|v|_{\infty} \leqslant c_{0}\left|v_{\xi}\right|$, with $c_{0}=\sqrt{L_{0}}>0$, and Young's inequality,

$$
\varepsilon^{2}|v|_{\infty}\left|v_{\xi}\right|\left|v_{\xi \xi}\right| \leqslant \varepsilon c_{0}\left|v_{\xi}\right|^{2}\left|v_{\xi \xi}\right| \leqslant \frac{v}{2}\left|v_{\xi \xi}\right|^{2}+\frac{1}{2 v} \varepsilon^{4} c_{0}^{2}\left|v_{\xi}\right|^{4}
$$

where $v>0$ can be chosen freely. Using again the Young inequality and the interpolation relation [Temam, Chap. III]

$$
\left|v_{\xi}\right|^{2} \leqslant \frac{v}{2}\left|v_{\xi \xi}\right|^{2}+\frac{1}{2 v}|v|^{2}
$$

it is easy to see that there are constants $c_{1}, c_{2}>0$, depending only on the norm of $V_{0}$ in $H^{2}$ (which controls $V(\tau)$ in $H^{2}$ and thereby $\left.\left|V_{\xi}\right|_{\infty}\right)$, but independent of $\varepsilon$ such that

$$
\frac{\mathrm{d}}{\mathrm{d} \tau}\left(|v|^{2}+\varepsilon\left|v_{\xi}\right|^{2}\right)+c_{2}\left|v_{\xi \xi}\right|^{2} \leqslant\left|V_{\tau}+V V_{\xi}\right|^{2}+c_{1}|v|^{2}+\varepsilon^{4}\left|v_{\xi}\right|^{4} .
$$

If $V_{\tau}+V V_{\xi}$ is square integrable in space and time, this differential inequality gives a bound on $A(\tau)=|v|^{2}+\varepsilon\left|v_{\xi}\right|^{2}$, which depends only on $L_{0}, T_{0}$ and $A(0)$, provided $\varepsilon$ is sufficiently small. To be precise, we have, in view of Section 2 , the following lemma.

Lemma 3.1 Let $V_{0} \in H^{2}$ and $v_{0} \in H^{1}$. There exists $\varepsilon_{0}>0$ such that, whenever $0<\varepsilon<\varepsilon_{0}$, $|v(\tau)|$ is uniformly bounded on the time interval $\left[0, T_{0}\right]$, independently of $\varepsilon$. The number $\varepsilon_{0}$ and the bound depend only on $V_{0} \in H^{2}, v_{0} \in H^{0}$ and $T_{0}$.

Combining with 3.47, which gives the control of the mean value of $u$, we obtain thanks to the Poincaré-Wirtinger inequality [Brezis]

THEOREM 3.2 Let $U_{0} \in H^{3}$ and $u_{0} \in H^{2}$. There exists $\varepsilon_{0}>0$ such that, whenever $0<\varepsilon<\varepsilon_{0}$, $|u(\xi, \tau)| \leqslant C$ for all $(\xi, \tau)$ in $\left[-L_{0} / 2, L_{0} / 2\right] \times\left[0, T_{0}\right]$. The number $\varepsilon_{0}$ and the bound $C$ depend only on $U_{0} \in H^{3}, u_{0} \in H^{1}$ and $T_{0}$.

Returning to the original problem, $\Phi=\varepsilon \varphi, \varphi=U+\varepsilon u$, we state the main result of the paper:

COROLlary 3.3 With the assumptions of Theorem 3.2 on the "well-prepared" initial data 3.48 , we have

$$
\max \left|\Phi(x, t)-\varepsilon U\left(x \sqrt{\varepsilon}, t \varepsilon^{2}\right)\right| \leqslant C \varepsilon^{2}
$$

for

$$
|x| \leqslant \frac{L_{0}}{2 \sqrt{\varepsilon}}, \quad 0 \leqslant t \leqslant \frac{T_{0}}{\varepsilon^{2}} .
$$

REMARK 3.4 The above result for the "well-prepared" initial data (3.48) combines in fact two results: one is the closeness to K-S proper, another one demonstrates continuity with respect to the initial conditions. In fact it would suffice to set $u_{0}(\xi)=0$ in (3.48), i.e. evolutions by two different equations are close if they start from the same initial configuration.

\section{Numerical simulation}

As was mentioned in the introduction, the objective of the numerical simulation was two-fold: firstly to verify closeness of the solutions of the quasi-steady model to the solutions of the K-S equations even for moderately small values of $\varepsilon$, and, secondly, to show that the quasi-steady model generates 
sufficiently rich dynamics including the occurrence of cellular structure and turbulence. We reiterate that the asymptotic result that was proved above does not by itself guarantee the asymptotic behavior of the solutions of the quasi-steady model until one shows existence of a global attractor and obtains appropriate estimates for the convergence to it.

Even then, it is not quite clear what kind of statements can be made regarding the structure of the attractor in comparison to those for the K-S equation. It is therefore desirable to carry out a more detailed numerical study of the dynamics generated by the quasi-steady model which we hope to discuss elsewhere in the near future.

For the numerical simulations it is more convenient to deal with the interval $[0, L]$ than $[-L / 2, L / 2]$. Thus, the periodic conditions are

$$
\Theta(0)=\Theta(L), \quad \Theta^{\prime}(0)=\Theta^{\prime}(L), \quad \Phi(0)=\Phi(L), \quad \Phi^{\prime}(0)=\Phi^{\prime}(L) .
$$

Next we need an explicit expression for the temperature $\Theta$ in spaces of periodic functions. Via variation of parameters and employing the boundary conditions it is uniquely defined as

$$
\begin{aligned}
\Theta(x) & =\alpha\left(I-\partial_{x}^{2}\right)^{-1} \Phi_{x x} \\
& =C_{1} e^{x}+C_{2} e^{-x}+\frac{\alpha}{2}\left(e^{-x} \int_{0}^{x} e^{\eta} \Phi_{\eta \eta} \mathrm{d} \eta-e^{x} \int_{0}^{x} e^{-\eta} \Phi_{\eta \eta} \mathrm{d} \eta\right)
\end{aligned}
$$

where

$$
C_{1}=\frac{\alpha}{2} \int_{0}^{L} \frac{e^{-\eta} \Phi_{\eta \eta}}{1-e^{-L}} \mathrm{~d} \eta, \quad C_{2}=\frac{\alpha}{2} \int_{0}^{L} \frac{e^{-(L-\eta)} \Phi_{\eta \eta}}{1-e^{-L}} \mathrm{~d} \eta .
$$

For convergence of the numerical code it is important to represent the expression for $\Theta$ with negative exponents under the integral. After some elementary manipulation it reduces to the following compact form:

$$
\Theta=\alpha \int_{0}^{L} \frac{e^{-\eta}+e^{-(L-\eta)}}{2\left(1-e^{-L}\right)} \Phi_{x x}(x-\eta) \mathrm{d} \eta
$$

As a result we obtain an integro-differential equation in terms of $\Phi$,

$$
\Phi_{\tau}+\frac{1}{2} \Phi_{x}^{2}=\Phi_{x x}-\alpha \int_{0}^{L} \frac{e^{-\eta}+e^{-(L-\eta)}}{2\left(1-e^{-L}\right)} \Phi_{x x}(x-\eta) \mathrm{d} \eta,
$$

which was integrated numerically.

Numerical solutions were carried out on the interval $L_{0}=14$ for the K-S equation, and for the appropriately rescaled (as dictated by the asymptotic result in the previous section) intervals $L=$ $L_{0} / \sqrt{\varepsilon}$ in the physical coordinate for the quasi-steady model. The time interval for the K-S equation was $T_{0}=20$, while the time intervals for the quasi-steady model were also adjusted in accordance with the theorem in Section 3. We set identical (appropriately rescaled) initial configurations.

The figures presented below do not exactly correspond to the actual evolution: an artificial drift $F(x, t):=\Phi(x, t)+.03 \times t$ was added for visualization purposes, in order to avoid overlapping of the fronts. One should keep in mind that the equations under discussion refer to a small perturbation of the basic front moving with constant velocity.

Figs. 1-3 show consecutive front positions generated by 4.59 ) for $\varepsilon=.25$ and $\varepsilon=.04$ (Figs. 1 and 2 respectively), and by the K-S equation (Fig. 3). For convenience the solution of the K-S is presented in the $x, t$ coordinates. 


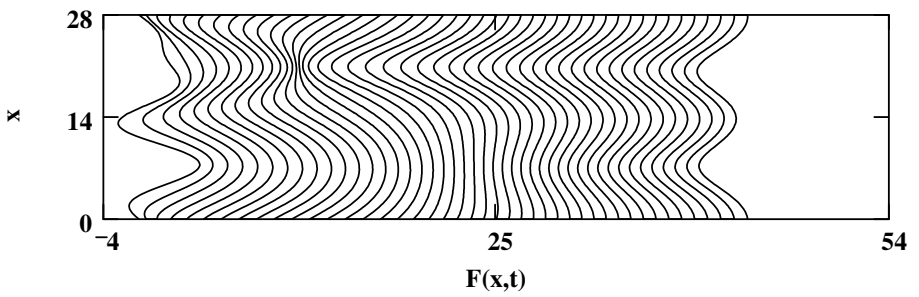

FIG. 1. Front evolution generated by 4.59 for $\varepsilon=.25$.

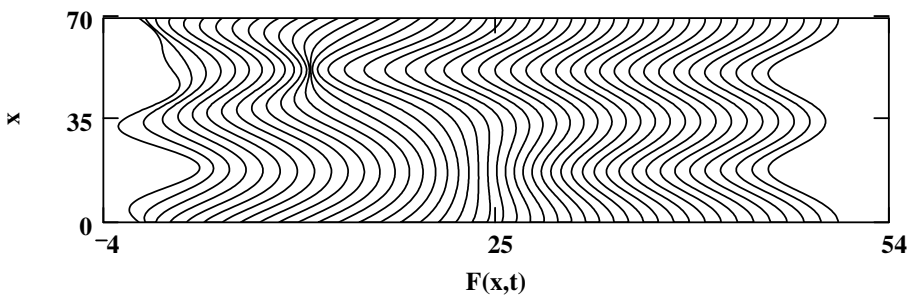

FIG. 2. Front evolution generated by 4.59 for $\varepsilon=.04$.

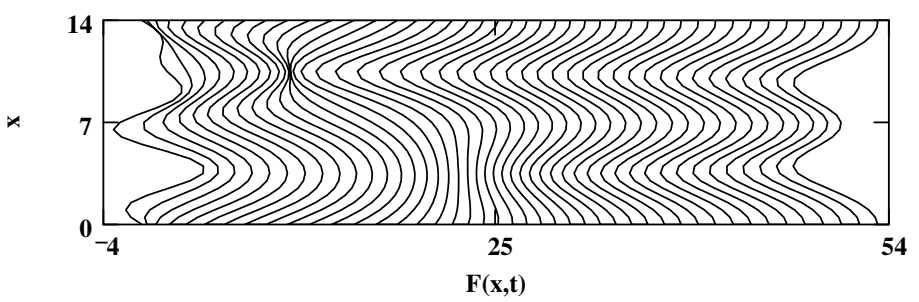

FIG. 3. Front evolution generated by the K-S equation.

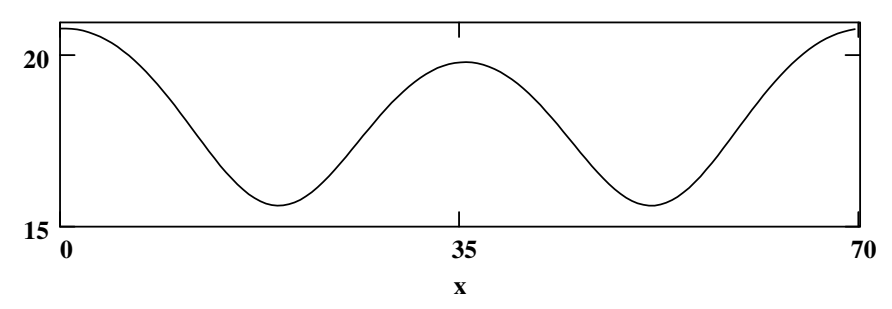

FIG. 4. A "two-cell" steady front configuration for $\varepsilon=.25$.

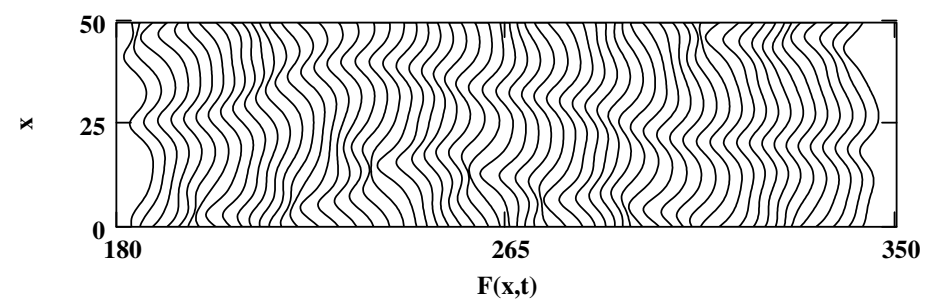

FIG. 5. Turbulent dynamics generated by the Q-S equation. 
Fig. 4 represents a single front generated by (4.59) for $\varepsilon=.25$ at the end of the time interval chosen for the simulation: $T=320$, which corresponds to this value of $\varepsilon$. We bring it here in order to give the reader a better idea of the true amplitude of the solutions.

Finally, Fig. 5 represents a fully developed turbulent regime generated by the quasi-steady model for $\alpha=1.25$, and a larger basic period $L=50$ (cf. Fig. 1). The evolution pattern shown occurs on a time interval $6000 \leqslant t \leqslant 8000$.

\section{Conclusions}

As was mentioned in the introduction, the advantage of the full $\kappa-\theta$ model or its quasi-steady truncation $1.5,1.6$ is that including the interface temperature may better reflect the properties of the original reaction-diffusion system (1.1), (1.3) while, at the same time, as we hope, they may serve as a basis for the description of the flame interaction with the background flow.

We have rigorously shown above that for the weakly nonlinear case, the periodic problem for the quasi-steady model is well-posed, and its solutions are asymptotically close to the solutions of the K-S equation in the sense of the instability parameter $\varepsilon$ for any fixed time interval.

On the other hand, we have demonstrated numerically a qualitative similarity of fully developed dynamical patterns between the Q-S equation and K-S equation, including the deeply nonlinear range where $\varepsilon$ is not small. Indeed, as we saw, the quasi-steady model is capable of generating cellular structures and turbulence. A more detailed numerical study that we intend to present in the near future shows a remarkable variety of dynamical patterns.

Since the full $\kappa-\theta$ model should be considered to be perhaps somewhat more physically sound, one faces the necessity to subject it to a similar study as the one presented above. We are hopeful if not convinced that, modulo overcoming some moderate technical difficulties, we shall be able to present results of such a study in the near future.

At the same time, we would like to reiterate that, although the Q-S equation was introduced as an ad hoc truncation of the full $\kappa-\theta$ model and the GI equation (1.4), it represents, in our opinion, an interesting dynamical system in its own right. It seems, so far, that the Q-S equation model is relatively "friendly" regarding the rigorous analysis, and we intend to demonstrate in the near future that its dynamics is essentially finite-dimensional in the sense of the attractor, inertial manifold, etc., once again similarly to the K-S equation.

Finally, we should remark that it would be interesting to understand what particular features of a nonlinear equation of a certain type are important for generation of specific dynamical patterns. At the same time, we would like to find out if and when such equations as for instance the Q-S equation and the K-S equation differ substantially in their qualitative behavior.

\section{Acknowledgements}

The work of C.M.B., J.H. and G.I.S. was supported in part by the RTN network "Nonlinear Partial Differential Equations describing Front Propagation and Other Singular Phenomena", HPRNCT-2002-00274. The collaboration between C.M.B. and J.H. was also supported by a CNRS PICS program. G.I.S. was also supported in part by The United States-Israel Binational Science Foundation, Grant 2002008; the German-Israel Foundation, Grant G-695-15.10.2001; and the Israel Science Foundation, Grant 67-01. M.F. was supported in part by the NSF Grant DMS-0207308 and CNRS. M.F., J.H and G.I.S. express their heartfelt gratitude to the University of Bordeaux 1 for its warm hospitality. 


\section{REFERENCES}

[Bhl01] Brauner, C.-M., Hulshof, J., \& Lunardi, A. A critical case of stability in a free boundary problem. J. Evolution Equations 1 (2001), 85-113. Zbl 0973.35036 MR 1838322

[BL00] BRAUNER, C.-M. \& LUNARDI, A. Instabilities in a two-dimensional combustion model with free boundary. Arch. Rat. Mech. Anal. 154 (2000), 157-182. Zbl 0984.76030 MR 1784964

[Brezis] BREZIS, H. Analyse Fonctionnelle, Théorie et Applications. Masson (1987). Zbl 0511.46001 MR 0697382

[DFM02] Di Francesco, M. \& MARCATI, P. Singular convergence to nonlinear diffusion waves for solutions to the Cauchy problem for the compressible Euler equations with damping. Math. Models Methods Appl. Sci. 12 (2002), 1317-1336. Zbl 1023.76040 MR 1927027

[FS87] Frankel, M. L. \& Sivashinsky, G. I. On the nonlinear thermal-diffusive theory of curved flames. J. Physique 48 (1987), 25-28.

[F88] Frankel, M. L. On the nonlinear evolution of solid-liquid interface. Phys. Lett. A 128 (1988), 57-60. MR 0935919

[FGS03] Frankel, M. L., Gordon, P. V., \& Sivashinsky, G. I. On desintegration of near-limit cellular flames. Phys. Lett. A $\mathbf{3 1 0}$ (2003), 389-392. MR 1976154

[GM97] GRENIER, E. \& MASMOUdi, N. Ekman layers of rotating fluids, the case of well prepared initial data, Comm. Partial Differential Equations 22 (1997), 953-975. MR 1976154

[MS79] MatKowsky, B. J. \& Sivashinsky, G. I. An asymptotic derivation of two models in flame theory associated with the constant density approximation. SIAM J. Appl. Math. 37 (1979), 686699. Zbl 0437.76065 MR 0549149

[NS84] Nicolaenko, B. \& Scheurer, G. Remarks on the Kuramoto-Sivashinsky equation. Phys. $D$ 12 (1984), 391-395. Zbl pre00576.35058 MR 0762813

[S80] Sivashinsky, G. I. On flame propagation under condition of stoichiometry. SIAM J. Appl. Math. 39 (1980), 67-82. Zbl 0464.76055 MR 0585829

[S83] Sivashinsky, G. I. Instabilities, pattern formation and turbulence in flames. Ann. Rev. Fluid Mech. 15 (1983), 179-199. Zbl 0538.76053

[Temam] Temam, R. Infinite-Dimensional Dynamical Systems in Mechanics and Physics. 2nd ed., Appl. Mat. Sci. 68, Springer (1997). Zbl 0871.35001 MR 1441312 